\title{
Familiarity with Diabetes as a Protective Factor for Severity of Condition at Type 1 Diabetes Diagnosis: The Need for More Secondary Prevention Methods in Public Health
}

\author{
TeriSue Smith-Jackson, PhD, MPH*, Matthew O. Flint, PhD, Mary V. Brown, PhD, Merilee \\ Larsen, DrPH \\ Department of Public and Community Health, Utah Valley University
}

\begin{abstract}
*Corresponding Author: TeriSue Smith-Jackson, PhD, MPH, Department of Public and Community Health, Utah Valley University, Email: Sue.Jackson@uvu.edu
\end{abstract}

\begin{abstract}
The purpose of this research was to explore the relationship between parental familiarity with type 1 diabetes and their child's condition at diagnosis. A 55-question online survey was completed by 975 parents of a minor child diagnosed with type 1 diabetes. Recruitment was completed through social media on groups specific to type 1 diabetes, as well as through snowball sampling. Results indicate that parents who had a high level of familiarity with type 1 diabetes signs and symptoms were more likely to have asked a health care provider to test the child for diabetes when symptoms occurred. Asking to have the child tested was statistically associated with several severity markers, including days spent in the hospital, time in an intensive care unit, diabetic ketoacidosis, and blood glucose at diagnosis. This data provides justification for the need for public health interventions aimed toward increasing familiarity of type 1 diabetes signs and symptoms in parents. Interventions for health promotion and education may include utilizing physicians, schools, news networks, and hospitals.
\end{abstract}

Keywords:Type 1 diabetes, secondary prevention, DKA prevention

\section{INTRODUCTION}

Primary prevention methods for type 2 diabetes are a frequent conversation in the field of public health. Type 2 diabetes is among one of the more preventable chronic health conditions, thus eliciting attention from public health professionals. Type 1 diabetes, on the other hand, is much less prevalent and has no primary prevention methods. Instead of having lifestyle causes, like many cases of type 2 , type 1 diabetes is an autoimmune disease in which the immune system attacks the body's beta cells that store and release insulin which prevents the body's ability to naturally regulate blood sugar levels. About 15,000 children are diagnosed with type 1 diabetes each year in the United States (Kaufman, Gallivan, \& Warren-Boulton, 2009), with incidence rates increasing (Choleau et al., 2014). It is primarily diagnosed during childhood, and has a sequential and predictable onset of symptoms (Insel et al., 2015). If not diagnosed promptly, type 1 diabetes can cause complications that can be life-threatening.

The onset of type 1 diabetes produces symptoms, such as excessive thirst, frequent urination, fatigue and weight loss, which are often confused with other conditions (Kaufman, Gallivan, \& Warren-Boulton, 2009). As a result, the diagnosis is often initially missed by parents and healthcare providers alike. At diagnosis, a child's pancreas is making little to no insulin, causing elevated blood glucose levels. If the diagnosis is delayed, the glucose levels will continue to increase, causing a more extreme condition of diabetic ketoacidosis (DKA), (Larsson et al., 2011; Pawlowicz, Birkholz, Neidzwiecki, \&Balcerska, 2009) which can lead to seizures, possibly resulting in permanent brain damage, and/or death. Previously published results have found that $34 \%$ of children with type 1 diabetes experienced a delayed diagnosis (Smith-Jackson, Brown, Flint, $\&$ Larsen, 2018).

The cause of type 1 diabetes is unknown; clinical researchers are working to determine primary prevention methods, but have been unsuccessful thus far. From a public health perspective, efforts focused on secondary prevention offer the most utility. There is currently not a uniform system for early 
detection and screening for type 1 diabetes in the United States. The increasing prevalence, in addition to the burden of disease on younger individuals, should emphasize the need for public health prevention measures.

The purpose of this research was to explore the relationship between parental familiarity with type 1 diabetes and their child's condition at diagnosis, as a means of justifying secondary prevention programs aimed at reducing severity of condition at diagnosis.

\section{METHODS}

\subsection{Participants}

Participants for this research study were the parent or guardian of a child with type 1 diabetes. The child had to have been diagnosed within the last ten years and be 18 years or younger at the time of the survey. A total of 1,352 individuals completed the electronic survey. Those who did not consent for participation were eliminated from the dataset, as were those who did not meet the inclusion criteria. For the purpose of this evaluation, only those from the United States were included in the analysis, leaving a total of 975 participants.

\subsection{Procedures and Measures}

University Institutional Review Board (IRB) approval was obtained prior to the implementation of this research study. No identifying information was collected from participants, and no incentives were used.

The 55-item survey was created by researchers to better understand the time period prior to the type 1 diabetes diagnosis, the actual diagnostic event, as well as the hospital stay that typically accompanies this diagnosis. Both qualitative and quantitative questions were used, with multiple choice, fill in the blank, and short answer options. The survey was pilot tested on a group from the target audience, and changes were made to reflect feedback given. The survey was conducted through Qualtrics, an online survey tool which allowed participants to complete the survey from any location or device.

As there is not a type 1 diabetes registry or database to construct a random sample, the most universal recruitment for participants was through snowball sampling targeting specific Facebook groups dedicated to type 1 diabetes education and support. A post by the researcher was made on several Facebook group pages related to type 1 diabetes with the survey link and a recruitment message. The post also asked participants to share the survey link to others who may be interested in completing the study and who would fit the inclusion criteria. The online survey was open for a one-week period in March 2016.

\subsection{Statistical Analysis}

Data was transferred from Qualtrics to an Excel file for cleaning. All participants who did not fit the inclusion criteria for this analysis and who did not consent to participate were eliminated. The data was cleaned and checked for accuracy before being imported into IBM SPSS (Version 21) [Computer software] for analysis.

\section{Results}

The survey participants were parents who had a child with type 1 diabetes. The parents were primarily white $(91.1 \%, \mathrm{n}=888)$ and female $(91.9 \%, \mathrm{n}=896)$, and had an average age of 40.46 years $(\mathrm{SD}=6.67, \mathrm{n}=958)$. Sixty percent of parents surveyed had earned a bachelor's degree or higher $(\mathrm{n}=586)$. Of the children diagnosed with type one diabetes, $54.1 \% \quad(n=527)$ were male and $45.8 \%(n=447)$ were female. The child's current age ranged from 1-18 years with an average of 9.67 years $(\mathrm{SD}=4.21, \mathrm{n}=975)$. All of the participants were diagnosed in the United States, and there was at least one participant from each of the 50 states and the District of Columbia. The majority of children $(74.4 \%$, $\mathrm{n}=725$ ) had private insurance at the time of diagnosis and $10.5 \%(n=102)$ had government insurance.

The mean age of the child at diagnosis in this sample was 6.49 years $(\mathrm{SD}=3.91)$. Prior to diagnosis, children were sick 19.13 days on average $(\mathrm{SD}=40.67)$. Parents reported most common symptoms prior to diagnosis as: frequent urination $(91.3 \%)$, increased thirst $(88.9 \%)$, weight loss (64.3\%), and fatigue $(61.5 \%)$. Parents were asked how familiar they were with type 1 diabetes prior to the diagnosis of their child, on a scale of $0-10$, with 0 being very unfamiliar and 10 being very familiar. The parents in this study had an average rating of $3.18(\mathrm{SD}=3.026, \mathrm{n}=975)$. Familiarity with type 1 diabetes was quite low for the majority of the parents prior to diagnosis. Almost two thirds $(63.5 \%, n=619)$ of the parents rated their familiarity with type 1 diabetes as a 3 or lower on the scale of $0-10$.

The parental familiarity of type 1 diabetes symptoms was associated with having 
Familiarity with Diabetes as a Protective Factor for Severity of Condition at Type 1 Diabetes Diagnosis: The Need for More Secondary Prevention Methods in Public Health

specifically asked to have the child checked for diabetes. Parents were asked if the adult present at the child's diagnosis asked for the child to be tested for type 1 diabetes. Overall, 38.2\% $(n=372)$ of respondents reported that an adult accompanying the child asked a health professional to test for diabetes, while $60.4 \%$ $(n=589)$ of respondents indicated that they did not specifically ask to be tested.

Among those who asked for a test, their average familiarity score was $4.12(\mathrm{SD}=3.19, \mathrm{n}=372)$, compared to a familiarity score of 2.57 $(\mathrm{SD}=2.748, \mathrm{n}=589)$ among those who did not ask for their child to be tested $(t=7.708$, $\mathrm{p}=<.001)$. As expected, those more familiar with type one diabetes were more likely to ask a Table1:Severity Indicators at Diagnosis healthcare provider for their child to be tested when symptoms occurred.

The health status at diagnosis for a child whose parent asked for the testing showed a dramatic difference in the severity outcomes. As shown in Table 1, when a parent asked to have the child tested at diagnosis, blood glucose level was lower, the perceived severity of the child's condition was better, and the child was transported by personal vehicle as opposed to ambulance or life flight to the hospital at diagnosis.

The child was also less likely to be in DKA, less likely to spend time in the Intensive Care Unit (ICU), and spent fewer total days in the hospital.

\begin{tabular}{|l|l|l|l|}
\hline & \multicolumn{1}{|c|}{ Asked for a test } & \multicolumn{1}{|c|}{ Did not ask for a test } & \multicolumn{1}{c|}{ Statistical value } \\
\hline Blood glucose $(\mathrm{mg} / \mathrm{dL})$ & $\mathrm{m}=547, \mathrm{n}=357$ & $\mathrm{~m}=606, \mathrm{n}=561$ & $\mathrm{t}=-3.98, \mathrm{p}<0.01$ \\
\hline DKA $(\%)$ & $25.5 \%, \mathrm{n}=95$ & $45 \%, \mathrm{n}=265$ & $\mathrm{x} 2=47.5, \mathrm{p}<0.01$ \\
\hline ICU $(\%)$ & $18.3 \%, \mathrm{n}=68$ & $34.6 \%, \mathrm{n}=204$ & $\mathrm{x} 2=39.1, \mathrm{p}<0.01$ \\
\hline Days in the hospital & $\mathrm{m}=3.28, \mathrm{n}=314$ & $\mathrm{~m}=4.02, \mathrm{n}=514$ & $\mathrm{t}=-2.3, \mathrm{p}=0.02$ \\
\hline Emergency transport & $14.6 \%, \mathrm{n}=46$ & $28.7 \%, \mathrm{n}=148$ & $\mathrm{x} 2=21.6, \mathrm{p}<0.01$ \\
\hline Severity at diagnosis & $\mathrm{m}=4.14, \mathrm{n}=372$ & $\mathrm{~m}=5.25, \mathrm{n}=585$ & $\mathrm{t}=-6.1, \mathrm{p}<0.01$ \\
\hline
\end{tabular}

\section{DISCUSSION}

Parental familiarity with type 1 diabetes is a powerful protective factor for earlier diagnosis and avoidance of DKA. In a parent questionnaire regarding the diagnosis of type 1 diabetes, children whose parents suspected a diabetes diagnosis were 1.3 times more likely to be diagnosed on the first visit to a healthcare professional (Usher-Smith, Thompson, Zhu, Sharp, \& Walter, 2015). It is well documented that children of families who do not have a close relative with diabetes are at greater risk of having DKA upon diagnosis (Choleau et al., 2014; Gunn et al., 2016; Klingensmith et al., 2013; Rewers, Dong, Slover, Klingensmith, $\&$ Rewers, 2015). This is likely because of a lack of parental familiarity with the symptoms of type 1 diabetes.

This research found that parents who were familiar with type 1 diabetes were more likely to ask their healthcare provider for a blood glucose test when they observed symptoms in their child that could be associated with type 1. To decrease the severity of complications at diagnosis, more secondary prevention work should be implemented to increase parental familiarity with the signs and symptoms associated with type 1 diabetes. When parents understand the symptoms of type 1 diabetes, they are more likely to request earlier testing, leading to better outcomes. While little work has been done in this regard in the United States, there have been several type 1 diabetes secondary prevention programs worldwide.

\subsection{A Global Look}

Earlier diagnosis and reduced rates of DKA upon diagnosis have been the goals of several successful type 1 diabetes awareness programs, primarily in Europe and Australia. One program launched in Parma, Italy reduced the rate of DKA upon diagnosis of type 1 diabetes from $78 \%$ to $12 \%$ within the span of seven years (Vanelli et al., 1999). This was accomplished through a multifaceted public awareness campaign targeting teachers, students, parents and pediatricians. Posters with practical information were posted at primary and secondary schools. Pediatricians were supplied with equipment to detect and information to diagnose type 1 diabetes early and rapidly. Lastly, a toll-free number was created to assist parents when diabetes was suspected(Vanelli et al., 1999).

In the United Kingdom (UK), "The 4 Ts" campaign, launched by a research charity Diabetes UK (2013), raised awareness about type 1 diabetes symptoms (Dowling, 2013). The 4 T's (tired, thirsty, thinner, and toilet) a mnemonic device was used to refer to the fatigue, excessive thirst, weight loss, and 
frequent urination associated with the disease. The program targeted parents and healthcare workers. After just one year, awareness of type 1 diabetes symptoms rose from $9 \%$ to $14 \%$ of the population being able to name all 4 symptoms. Parents were taught to take their child to the doctor if one or more symptoms are present, and to insist on a test (Dowling, 2013). The mnemonic of the 4 T's has been used in education programs in both India and Australia as well ("The 4 T's of type," 2015). The organization, Diabetes Australia (n.d.), used the 4 T's in launching a campaign in 2017 called, "It's About Time". The program urged communities, families, schools and health professionals to recognize the symptoms of type 1 diabetes earlier.

Also in the UK, the EDDY study (The Early Detection of Type 1 Diabetes in Youth) made reusable shopping bags with the diabetes symptoms on the side. The bag, which also contained a brochure with further information, was sent home with school-age children. The campaign also provided primary health care professionals with materials to check glucose levels, and educational leaflets (Townson et al., 2017).

In Finland, researchers screened children at birth for genetic type 1 diabetes risk. Among Finnish families whose child tested positive for increased risk at birth and who received followup education, they had only a 5\% rate of DKA upon diagnosis. Children who screened positive and did not receive follow-up education had a 23\% rate of DKA upon diagnosis (Hekkala, Homen, Toppari, Knip, \&Veijola, 2017).

\subsection{Implications for Practice}

In the United States, individual researchers and non-profit organizations have done work to educate about the signs and symptoms of type 1 diabetes, but there are no national coordinated efforts, or even large-scale intervention programs. Several public health programs could be initiated at local, state and national levels to help increase parental familiarity with type 1 diabetes in the United States. Some ideas could include

- Adding information about type 1 diabetes to educate parents or caregivers at well-child visits at primary care doctors and pediatricians. This information could be distributed through fliers, posters, electronic media and/or handouts throughout medical offices. Even something as simple as a clear and memorable poster, highlighting the symptoms of type 1 diabetes hanging in a patient waiting room could increase parental familiarity.

- Teacher education could provide another effective avenue to increase awareness of type 1 symptoms. In many elementary classrooms, due to the time teachers consistently spend with their students, the teacher may be more aware of changes in a student's fluid intake and bathroom habits than even the child's parents. There is little by way of teacher education in schools to increase awareness of the symptoms of type 1 diabetes for early diagnosis as most interventions concerning type 1 in schools are focused on treatment of existing cases (Pansier\& Schulz, 2013). Educating teachers to recognize and understand the signs and symptoms, along with reminders through marketing tools, posters, emails and teacher trainings could help increase awareness and encourage teachers to contact parents should symptoms arise. This type of training could be provided for pre-service teachers in universities, at preschools and daycares with child caregivers, at sports facilities with coaches and camp locations with all camp staff as well.

- Marketing posters could also be placed where families traditionally gather or places where parents or caregivers congregate. Places such as city libraries, grocery stores, church buildings, gas stations, on busses, and at local recreation centers. Messaging about type 1 diabetes could also give information about where to go locally to get your child tested, thus feeding correct information into specific communities.

- Traditional news and social media networks could be utilized to share public service announcements, news stories, and information related to type 1 diabetes. Clear, consistent messaging of type 1 signs and symptoms is needed.

- Hospitals and insurance companies could be utilized to share awareness and educational messages. Posters displayed on hospital walls, or public service announcement messages on hospital televisions could increase awareness among a general population. 


\subsection{Limitations and Future Research}

Not having a universal database for those with type 1 diabetes limited the methodology options, leading to snowball sampling through social media. This sampling limitation may limit the findings. The participants in this research were primarily white, and had young children with type 1 diabetes, which may be a reflection of social media usage. The data may also be influenced by volunteer bias, as those with more negative experiences may have been more likely to complete the survey.

Future research should include developing and evaluating interventions, as proposed earlier. Having quality data on the effectiveness of these approaches will be essential in getting long-term funding for secondary prevention programs.

\section{CONCLuSion}

Results indicate that increased parental familiarity with signs and symptoms of type 1 diabetes translates into an increased likelihood to ask to have the child tested when symptoms occur. Asking to have the child tested was statistically associated with several severity markers, including days spent in the hospital, DKA, and blood glucose at diagnosis. This data provides justification for the need for health promotion and education activities geared toward increasing familiarity of parents with type 1 diabetes. Various public health strategies, including utilizing physicians, schools, news networks, and hospitals could be employed in this regard.

\section{ACKNOWLEDGEMENTS}

The researchers would like to acknowledge the student assistants who helped with this project: Sarah Waddoups, Sarah Callaway, Stacy Mickelson, Kami Hartley, Dakota Froisland, and Karen Spong.

\section{REFERENCES}

[1] Choleau, C., Maitre, J., FilipovicPierucci, A., Elie, C., Barat, P., Bertrand, A., \& ... Robert, J. (2014). Ketoacidosis at diagnosis of type 1 diabetes in French children and adolescents. Diabetes \& Metabolism, 40(2), 137-142. doi: 10.1016/j.diabet.2013.11.001

[2] Diabetes Australia. (n.d.). 'It's About Time' we detected all types of diabetes earlier. Retrieved from

https://static.diabetesaustralia.com.au/s/fileasset s/diabetes-australia/904c3fd5-8af6-4a07-b4302e2123215df6.pdf
[3] Diabetes UK. (2013). Significant increase in parents' awareness of Type 1 diabetes symptoms. Retrieved from https://www.diabetes.org.uk/About_us/News/Si gnificant-increase-in-parents-awareness-ofType-1-diabetes-symptoms/

[4] Dowling, L. (2013). The 4 'Ts' - aiding prompt diagnosis of type 1 diabetes in children. Practice Nurse, 43(2), 18-21.

[5] Gunn, E. R., Albert, B. B., Hofman, P. L., Cutfield, W. S., Gunn, A. J., \& Jefferies, C. A. (2016). Pathways to reduce diabetic ketoacidosis with new onset type 1 diabetes: Evidence from a regional pediatric diabetes center: Auckland, New Zealand, 2010 to 2014. Pediatric Diabetes, 18(7), 553-558. doi:10.1111/pedi.12456

[6] Hekkala, A. M., Ilonen, J., Toppari, J., Knip, M., \&Veijola, R. (2017). Ketoacidosis at diagnosis of type 1 diabetes: Effect of prospective studies with newborn genetic screening and follow up of risk children. Pediatric Diabetes, 19(2), 314-319. doi:10.1111/pedi.12541

[7] IBM Corp. Released 2012: IBM SPSS Statistics for Windows, Version 21.0. Armonk, NY: IBM Corp.

[8] Insel, R. A., Dunne, J. L., Atkinson, M. A., Chiang, J. L., Dabelea, D., Gottlieb, P. A., Ziegler, A. G. (2015). Staging presymptomatic type 1 diabetes: A scientific statement of JDRF, the Endocrine Society, and the American Diabetes Association. Diabetes Care, 38(10), 1964-1974. doi:10.2337/dc15-1419

[9] Kaufman, F. R., Gallivan, J. M., WarrenBoulton, E. (2009). Overview of diabetes in children and teens. American Journal of Health Education, 40(5), 259-263.

[10] Klingensmith, G. J., Tamborlane, W. V., Wood, J., Haller, M. J., Silverstein, J., Cengiz, E., \& ... Beck, R. W. (2013). Diabetic ketoacidosis at diabetes onset: still an all too common threat in youth. The Journal of Pediatrics, 162(2), 330334. doi: 10.1016/j.jpeds.2012.06.058

[11] Larsson, H. E., Vehik, K., Bell R, Dabelea, D., Dolan, L., Pihoker, C., Haller, M. J. (2011). Reduced prevalence of diabetic ketoacidosis at diagnosis of type 1 diabetes in young children participating in longitudinal follow-up. Diabetes Care, 34(11), 2347-2352. doi:10.2337/dc11-1026

[12] Pansier, B., \& Schulz, P. J. (2015). Schoolbased diabetes interventions and their outcomes: A systematic literature review. Journal of Public Health Research, 4(1), 6571. doi:10.4081/jphr.2015.467

[13] Pawlowicz, M., Birkholz, D., Niedzwiecki, M., \&Balcerska, A. (2009). Difficulties or mistakes in diagnosing type 1 diabetes in children? -Demographic factors influencing delayed 
diagnosis. Pediatric Diabetes, 10(8), 542-549. doi:10.1111/j.1399-5448.2009. 00516.x

[14] Rewers, A., Dong, F., Slover, R. H., Klingensmith, G.J., \&Rewers, M. (2015). Incidence of diabetic ketoacidosis at diagnosis of type 1 diabetes in Colorado youth, 19982012. JAMA, 313(15),1570-1572. doi:10.1001/ jama.2015.1414

[15] Smith-Jackson, T., Brown, M. V., Flint, M., \& Larsen, M. (2018). A mixed method approach to understanding the factors surrounding delayed diagnosis of type one diabetes. Journal of Diabetes and its Complications, 32(11), 1051-1055. doi: 10.1016/j.jdiacomp.2018.08. 011

[16] The 4 T's of type 1 diabetes. (2015, September 23), The Bombay News: E Times. Retrieved from https://timesofindia.indiatimes.com/ lifes pan-news/The-4-Ts-of-type-1-diabetes/article show/49071828.cms
[17] Townson, J., Gregory, J. W., Cowley, L., Gallagher, D., Channon, S., Robling, D., ... Lowes, L. (2017). Establishing the feasibility of a community and primary health care intervention to raise awareness of symptoms of type 1 in youth (EDDY) study. Pediatric Diabetes, 18(8), 955-963. doi:10.1111/pedi/ 12516

[18] Usher-Smith, J. A., Thompson, M. J., Zhu, H., Sharp, S. J., \& Walter, F. M. (2015). The pathway to diagnosis of type 1 diabetes in children: a questionnaire study. BMJ Open, 5, 1-10. doi:10.1136/bmjopen-2014-006470

[19] Vanelli, M., Chiari, G., Ghizzoni, L., Costi, G., Giacalone, T., \&Chiarelli, F. (1999). Effectiveness of a prevention program for diabetic ketoacidosis in children. An 8-year study in schools and private practices. Diabetes Care, 22(1), 7-9. doi:10.2337/diacare.22.1.7.

Citation: TeriSue Smith-Jackson, PhD, MPH, Matthew O. Flint, et.al Familiarity with Diabetes as a Protective Factor for Severity of Condition at Type 1 Diabetes Diagnosis: The Need for More Secondary Prevention Methods in Public Health. ARC Journal of Diabetes and Endocrinology. 2019; 5(1):29-34. doi:dx.doi.org/10.20431/2455-5983.0501004.

Copyright:(C) 2019 Authors. This is an open-access article distributed under the terms of the Creative Commons Attribution License, which permits unrestricted use, distribution, and reproduction in any medium, provided the original author and source are credited. 